\title{
Dosagem sérica de troponina I em cães com desnível do segmento ST utilizando quimioluminescência
}

\author{
[Serum determination of troponin I in dogs with ST deviation \\ by chemiluminescent] \\ A.L.F. Santos ${ }^{1}$, M.H.M.A. Larsson $^{1}$, G.G. Pereira ${ }^{1}$, M.M. Santos ${ }^{1}$, V.C.R. Gutierrez ${ }^{2}$ \\ ${ }^{1}$ Faculdade de Medicina Veterinária e Zootecnia - Universidade de São Paulo \\ Av. Prof. Orlando Marques de Paiva, 87 \\ 05508-270 - São Paulo, SP \\ ${ }^{2}$ Hospital Universitário - Universidade de São Paulo - São Paulo, SP
}

\begin{abstract}
RESUMO
Com o intuito de verificar algum dano nas células do miocárdio, utilizaram-se 38 cães, 20 com traçado eletrocardiográfico normal, grupo 1, e 18 com desníveis do segmento ST, grupo 2, em registro na derivação II, velocidade de $50 \mathrm{~mm} / \mathrm{s}$ e sensibilidade $\mathrm{N}(1 \mathrm{mV}=1 \mathrm{~cm})$. No grupo 1 , a dosagem sérica da troponina I (cTnI) destinou-se à obtenção dos valores referenciais $(\mathrm{ng} / \mathrm{mL})$ que seriam confrontados com os obtidos no grupo 2. A média e o desvio-padrão foram, respectivamente, $0,16 \mathrm{ng} / \mathrm{mL}$ e $0,11 \mathrm{ng} / \mathrm{mL}$ e $0,20 \mathrm{ng} / \mathrm{mL}$ e $0,11 \mathrm{ng} / \mathrm{mL}$, nos grupos 1 e 2 . A cTnI não apresentou evidências de associação com idade, massa corpórea, creatinafosfoquinase total e potássio nos dois grupos. Não houve diferenças significativas nos valores de cTnI entre os grupos. Conclui-se que é possível a utilização do kit de ensaio imunométrico quimioluminescente humano para a espécie canina e que a hipóxia-isquemia, revelada pelo desnível do segmento ST não acarreta dano miocárdico ou este é mínimo e indetectável.
\end{abstract}

Palavras-chave: cão, troponina I, segmento ST, eletrocardiografia

\begin{abstract}
In order to investigate myocardial cells injury, 38 dogs were evaluated, being 20 with a normal electrocardiogram (group 1) and 18 with ST segment elevation or depression (group 2), recorded in lead II, at paper speed of $50 \mathrm{~mm} / \mathrm{sec}$ and $N$ sensibility $(1 \mathrm{mV}=1 \mathrm{~cm})$. Serum measurement of troponin I (cTnI) in group 1 was determined to obtain reference values $(\mathrm{ng} / \mathrm{mL})$. These values were compared to those obtained in dogs from group 2, to confirm or not myocardial injury. Mean cTnI values in groups 1 and 2 were $0.16 \mathrm{ng} / \mathrm{mL}(S D \pm 0.11 \mathrm{ng} / \mathrm{mL})$ and $0.20 \mathrm{ng} / \mathrm{mL}(S D \pm 0.11 \mathrm{ng} / \mathrm{mL})$, respectively. Three cTnI null values were found in group 1. cTnI was not related to age, mass, CK-T or serum potassium concentration in both animal groups, for each level varied in the group. There was no difference in cTnI values between groups 1 and 2. In conclusion, it is possible to use the human chemiluminescent immunometric assay kit in canine species and hypoxia/ischemia revealed by ST segment deviation does not mean significant myocardium injury.
\end{abstract}

Keywords: dog, troponin I, Dogs, ST segment, electrocardiography

\section{INTRODUÇÃO}

O infarto agudo do miocárdio (IAM) é uma afecção comum no homem, cujo sintoma típico é a "dor no peito", irradiada para braços e costas. Indivíduos com estes sintomas são monitorados

Recebido em 4 de junho de 2010

Aceito em 30 de junho de 2011

E-mail: andrvet@usp.br pelo exame eletrocardiográfico, bem como por meio da dosagem dos marcadores de hipóxiaisquemia miocárdica (Bonow et al., 2009). Entende-se por hipóxia miocárdica a insuficiente oxigenação do músculo cardíaco (Robbins et al., 2001), apesar da perfusão adequada (Bonow et al., 2009), enquanto isquemia é a privação de 
oxigênio, acompanhada de inadequada remoção dos metabólitos.

Um marcador de injúria miocárdica muito utilizado são as troponinas. O complexo troponina regula a interação actina-miosina nos músculos estriados (esquelético e cardíaco) e tem, portanto, papel no acoplamento eletromecânico dessa musculatura, não sendo encontrado na musculatura lisa (Lobetti et al., 2002; Schobber et al., 2002a; Procajlo et al., 2005; Fonfara et al., 2010).

A liberação de troponina na circulação sanguínea ocorre pela dissociação do aparelho contrátil e relaciona-se à lesão irreversível na célula miocárdica, apresentando, na circulação, meiavida de duas horas e excreção renal (Ramos e Magalhães, 2000). Sabe-se, segundo Feng et al. (1998), que não há aumento nos níveis séricos de troponina I (cTnI) em indivíduos que apresentem doença muscular crônica. A estrutura das troponinas cardíacas é semelhante no cão e no homem (Schobber et al., 2002b). Os genes que codificam cTnI foram sequenciados e clonados nas espécies canina e felina e observou-se semelhança de 95 e $96 \%$, respectivamente, em relação aos mesmos genes no homem (Rishniw et al., 2004). Esse fato ratifica o uso de imunoensaios, utilizando anticorpo monoclonal humano, no diagnóstico de lesão cardíaca em cães e gatos (Rishniw et al., 2004; Adin et al., 2006; Burgener et al., 2006; Fonfara et al., 2010). Nos últimos anos, desenvolveram-se métodos de ensaios analíticos baseados no princípio da quimioluminescência, que ocorre na oxidação de certas moléculas (Weeks, 1997). A oxidação gera produtos em estado eletrônico excitado e estes, consequentemente, emitem luz (Weeks, 1997; Feitosa et al., 2002).

A aterosclerose é rara nos cães e, quando ocorre, está associada ao hipotireoidismo não controlado, porém hipóxia-isquemia, com consequente infarto, é comum em decorrência de embolismo coronariano - endocardite infecciosa, particularmente da valva aórtica -, septicemia e neoplasia pulmonar (Thomas, 1987). Cães que apresentam a síndrome dilatação-torção gástrica frequentemente desenvolvem arritmias ventriculares - extra-sístoles e taquicardia ventricular -, que podem ocasionar isquemia focal e necrose miocárdica, e o mesmo fato ocorre na pancreatite aguda (Fox, 1992; Herndon et al., 2002; Burgener et al., 2006).

Cardiopatias que evoluem para insuficiência cardíaca congestiva - cardiomiopatias dilatada, hipertrófica, restritiva e endocardiose mitral e/ou tricúspide - causam hipóxia-isquemia do músculo cardíaco e, consequentemente, induzem à liberação de cTnI na circulação (Schobber et al., 2002a; Herndon et al., 2002; Fonfara et al., 2010). Além da detecção do dano miocárdico, a cTnI pode ser utilizada para monitorar o dano miocárdico cronicamente, servindo como parâmetro prognóstico (Fonfara et al., 2010). O segmento ST pode apresentar duas alterações em sua morfologia: o infra e o supradesnível, indicando má oxigenação do miocárdio (Tilley, 1992).

Os distúrbios eletrolíticos, especialmente os relacionados ao cátion potássio $(\mathrm{K})$, podem causar alterações na morfologia do segmento ST (Tilley, 1992). A hipopotassemia ocorre quando o nível sérico de potássio está abaixo de 3,0mEq/L, ocasionando aumento na relação $\mathrm{K}$ intracelular: $\mathrm{K}$ extracelular $\mathrm{e}$ tornando $\mathrm{O}$ miocárdio menos excitável. Tem como causas a insuficiência renal crônica, a administração excessiva de mineralocorticoides, a emese e quadros diarreicos severos e a administração, de altas doses e por período prolongado, dos diuréticos de alça (Tilley e Junior, 2008). Na hiperpotassemia, a concentração sérica de potássio ultrapassa os $6,5 \mathrm{mEq} / \mathrm{L}$ e há, consequentemente, a diminuição na relação $\mathrm{K}$ intracelular/K extracelular e hiperexcitabilidade miocárdica, tendo como causas a insuficiência renal aguda, ruptura vesical, obstrução uretral, trauma muscular, intoxicação digitálica, altas dosagens de diuréticos poupadores de potássio, entre outras (Tilley e Junior, 2008).

\section{MATERIAL E MÉTODOS}

Foram utilizados 38 cães, machos ou fêmeas, com ou sem definição racial, pesos corpóreos e idades variadas, distribuídos em dois grupos: o grupo 1 foi constituído por 20 cães hígidos e com exames eletrocardiográficos normais, e o grupo 2 por 18 cães que apresentavam desníveis (infra ou supra) do segmento ST.

Os cães utilizados foram provenientes do Serviço de Cardiologia de Hospital Veterinário Escola e 
seu uso foi aprovado pela Comissão de Bioética da Faculdade de Medicina Veterinária e Zootecnia da USP, protocolo $n^{\circ}$ 238/2002. Cães sabidamente portadores de neoplasias, gastroenterite, dermatopatias, miopatias e frequentadores das regiões litorâneas, em razão do risco de contraírem a Dirofilaria immitis, não foram incluídos no estudo. $\mathrm{O}$ exame físico consistiu na determinação das frequências cardíaca e respiratória, mensuração da temperatura retal, inspeção das mucosas oral, ocular e peniana-vaginal, palpação abdominal e dos linfonodos e auscultação cardiorrespiratória.

Os exames radiográficos foram realizados no aparelho de radiodiagnóstico CGR, modelo Chenonceaux, de $600 \mathrm{~mA}$ e $130 \mathrm{Kv}$, com mesa radiológica com grade e sistema Potter-Buck recipromático tipo Par Speed, ampola de raios-X de anodo giratório. Os filmes foram revelados e fixados na processadora automática RP-OMAT Processor Kodak. O exame radiográfico foi realizado em três projeções: laterolateral direita, laterolateral esquerda e ventrodorsal.

Os exames eletrocardiográficos foram realizados em eletrocardiógrafo Ecafix modelo ECG-6 ou Schiller modelo AT-1v, com os animais posicionados em decúbito lateral direito, sobre superfície isolante. Realizaram-se as derivações bipolares $\mathrm{D}_{\mathrm{I}}, \mathrm{D}_{\mathrm{II}}, \mathrm{D}_{\mathrm{III}}$ e as unipolares aumentadas aVR, aVL e aVF, além das derivações précordiais $\mathrm{CV}_{5} \mathrm{RL}, \mathrm{CV}_{6} \mathrm{LL}, \mathrm{CV}_{6} \mathrm{LU}$ e $\mathrm{V}_{10}$, segundo Tilley e Junior (2008).

Os exames ecocardiográficos foram realizados em aparelho de ultrassonografia Hitachi modelo EUB 515-A, com transdutor de $5 \mathrm{MHz}$, microconvexo. A impressora utilizada foi a SONY modelo UP 890-CE. Foram mensurados e calculados os seguintes parâmetros no modo M, segundo as especificações e os valores de referência de Bonagura (1983), Boon (1998) e Moise e Fox (1999): dimensão da cavidade do ventrículo esquerdo no final da sístole (DSVEs); espessura da parede livre do ventrículo esquerdo na diástole (Epd); espessura do septo interventricular em diástole (ESd); fração de encurtamento (FE), calculada por meio da fórmula (DVEd-DVEs)x100/DVEd; raiz da aorta (Ao); diâmetro do átrio esquerdo (AE) e relação entre Ao e AE.
As amostras de sangue foram colhidas por meio da punção das veias radial ou safenas ou jugulares - direitas ou esquerdas -, utilizando agulhas e seringas descartáveis e tubos siliconizados, providos de rolha de borracha (Vacutainer®), com capacidade para $4 \mathrm{~mL}$. Utilizaram-se tubos contendo EDTA-K2 $(3,6 \mathrm{mg})$ com anticoagulante, para a realização do hemograma, e tubos sem anticoagulante, para os exames de bioquímica sérica e cTnI. Após a realização da colheita $-10 \mathrm{~mL}$ de cada animal - , o sangue foi centrifugado a $1500 \mathrm{~g}$, durante 10 minutos. O soro para dosagem de cTnI foi armazenado a $-20^{\circ} \mathrm{C}$ até, no máximo, 30 dias. A dosagem sérica de potássio foi efetuada em, no máximo, 24 horas, e o armazenamento foi idêntico ao das amostras para a dosagem de CKMB (massa) e cTnI.

Hemograma, perfis renal e hepático e determinação sérica de potássio foram realizados em Laboratório Clínico Escola, enquanto a mensuração da cTnI foi realizada em Laboratório Clínico de Hospital Universitário Escola. A contagem global de hemácias e leucócitos e a determinação da hemoglobina foram realizadas utilizando-se o sistema automatizado Serono modelo System 9020 AX. O hematócrito foi determinado pela técnica do micro-hematócrito, em centrífuga Celm modelo $\mathrm{MH}$, e a contagem diferencial dos leucócitos realizada ao microscópio óptico Carl Zeiss Jenamed, em esfregaços de sangue in natura, corados pelo Rosenfeld.

A avaliação dos perfis renal e hepático foi realizada no analisador bioquímico automático Technicon-Bayer, modelo RA-100. O perfil renal foi avaliado por meio das dosagens séricas de ureia - método colorimétrico da urease - e de creatinina, pelo método cinético. O perfil hepático foi avaliado por meio da determinação sérica da fosfatase alcalina método cinético colorimétrico -, aspartato e alanino aminotransferases - método cinético em ultra-violeta -, proteína total e albumina.

A dosagem de potássio sérico foi realizada pela técnica do eletrodo íon seletivo, utilizando-se o analisador OMNI-4. 
Os procedimentos do ensaio para cTnI (Diagnostic ..., 2002) foram: 1 - adição de $50 \mu \mathrm{L}$ do soro do paciente na unidade teste, onde há a pérola revestida com anticorpo monoclonal de rato antitroponina e incubação a $37^{\circ} \mathrm{C}$, durante 30 minutos, sob agitação intermitente; 2 - ligação da cTnI ao anticorpo da pérola; 3 - remoção do restante do soro, por centrifugação, para a porção coaxial da unidade teste; 4 - adição de $6,5 \mathrm{~mL}$ de fosfatase alcalina de intestinos de vitelo, conjugada ao anticorpo policlonal de cabra antitroponina I tamponizada; 5 - adição do substrato quimioluminescente (éster fosfato adamantil dioxetano), incubação por 10 minutos e realização da leitura no luminômetro; 6 - para algumas características do ensaio: a) calibração de até $180 \mathrm{ng} / \mathrm{mL}$; b) sensibilidade analítica de $0,1 \mathrm{ng} / \mathrm{mL}$; c) específicidade para cTnI; d) bilirrubina no soro - causa de depressão nos valores mensurados; e) hemólise de até $570 \mathrm{ng} / \mathrm{mL}$ e lipemia de até $5000 \mathrm{ng} / \mathrm{mL}$ - que não alteram a precisão do ensaio.

\section{RESULTADOS}

Os animais do grupo 1 apresentaram hemograma, perfil renal, perfil hepático e exames radiográficos do tórax dentro dos padrões de normalidade (Meyer e Harvey, 1998; Tilley e Junior, 2008).

Os cães dos grupos 1 e 2 apresentaram disparidade em relação à faixa etária e peso corpóreo. Os do grupo 1 eram mais jovens -19 cães com idade abaixo de seis anos. No grupo 2 prevaleceram cães mais velhos, com 15 animais com idade acima de sete anos (Tab. 1). Os cães do grupo 1 apresentaram massa corpórea maior e com distribuição mais homogênea, em relação aos do grupo 2 (Tab. 2). Quanto aos níveis séricos de cTnI, para os do grupo 1, a média e o desvio-padrão foram, respectivamente, de $0,16 \mathrm{ng} / \mathrm{mL}$ e $0,11 \mathrm{ng} / \mathrm{mL}$, enquanto para o grupo 2 foram, respectivamente, de $0,20 \mathrm{ng} / \mathrm{mL}$ e $0,11 \mathrm{ng} / \mathrm{mL}$. O teste exato de Fisher, a $5 \%$ de significância, não detectou associação entre os grupos e o nível sérico de cTnI.

Tabela 1. Identificação e concentração séria de cTnI nos animais sem alterações eletrocardiográficas (grupo 1)

\begin{tabular}{llllll}
\hline Número & $\begin{array}{l}\text { Definição } \\
\text { racial }\end{array}$ & Sexo & $\begin{array}{l}\text { Idade } \\
(\text { anos })\end{array}$ & $\begin{array}{l}\text { Massa } \\
(\mathrm{kg})\end{array}$ & $\begin{array}{l}\text { cTnI } \\
(\mathrm{ng} / \mathrm{mL})\end{array}$ \\
\hline 1 & SRD & F & 2 & 14,70 & 0,28 \\
2 & SRD & M & 2 & 6,20 & 0,07 \\
3 & SRD & F & 3 & 20,40 & $<0,20$ \\
4 & SRD & F & 2 & 10,30 & $<0,20$ \\
5 & SRD & M & 6 & 21,30 & 0,07 \\
6 & Poodle toy & F & 6 & 7,00 & 0,43 \\
7 & Poodle toy & F & 6 & 4,20 & 0,27 \\
8 & SRD & M & 2 & 10,20 & $<0,20$ \\
9 & Poodle toy & M & 6 & 8,20 & 0,16 \\
10 & Labrador & F & 3 & 31,80 & 0,05 \\
11 & Pastor Belga & M & 7 & 24,20 & 0,15 \\
12 & Teckel & M & 2 & 12,40 & 0,15 \\
13 & SRD & F & 3 & 24,70 & 0,12 \\
14 & SRD & M & 5 & 19,00 & 0,22 \\
15 & SRD & F & 2 & 21,80 & 0,09 \\
16 & SRD & M & 3 & 16,60 & 0,19 \\
17 & SRD & M & 3 & 19,00 & 0,24 \\
18 & SRD & M & 2 & 12,00 & 0,23 \\
19 & SRD & M & 2 & 17,40 & 0,23 \\
20 & SRD & M & 3 & 15,40 & 0,17 \\
\hline SRD: & S & Fem & & & \\
\hline
\end{tabular}

SRD: sem raça definida; M: masculino; F: feminino. 
Tabela 2. Identificação e concentração sérica de cTnI nos animais com desníveis do segmento ST (grupo 2)

\begin{tabular}{llllll} 
Número & $\begin{array}{l}\text { Definição } \\
\text { racial }\end{array}$ & Sexo & $\begin{array}{l}\text { Idade } \\
\text { (anos) }\end{array}$ & $\begin{array}{l}\text { Massa } \\
\text { (kg) }\end{array}$ & $\begin{array}{l}\text { cTnI } \\
(\mathrm{ng} / \mathrm{mL})\end{array}$ \\
\hline 1 & Poodle & M & 10 & 7,7 & 0,13 \\
2 & SRD & M & 16 & 5,9 & 0,03 \\
3 & Cocker Spaniel & F & 3 & 10,0 & 0,24 \\
4 & Poodle toy & M & 8 & 2,8 & 0,52 \\
5 & Dobermann & M & 9 & 26,0 & 0,33 \\
6 & Poodle & M & 12 & 15,2 & 0,26 \\
7 & Yorkshire & F & 9 & 3,2 & 0,24 \\
8 & Poodle & M & 8 & 10,7 & 0,15 \\
9 & Terrier Brasileiro & F & 11 & 15,3 & 0,05 \\
10 & Poodle & M & 14 & 4,8 & 0,22 \\
11 & Teckel & M & 11 & 5,6 & 0,20 \\
12 & Poodle toy & M & 11 & 2,6 & 0,18 \\
13 & Poodle toy & F & 1 & 2,4 & 0,14 \\
14 & Terrier Brasileiro & M & 4 & 11,4 & 0,31 \\
15 & Cocker Spaniel & F & 11 & 14,0 & 0,15 \\
16 & SRD & F & 12 & 10,8 & 0,15 \\
17 & Teckel & M & 9 & 11,2 & 0,14 \\
18 & Cocker Spaniel & F & 7 & 12,0 & 0,19 \\
\hline
\end{tabular}

SRD: sem raça definida; M: masculino; F: feminino.

\section{DISCUSSÃO}

A literatura médico-veterinária ainda é escassa em trabalhos que contemplem os marcadores cardíacos, porém nos últimos anos houve a publicação de alguns artigos que mensuraram as troponinas $\mathrm{T}$ e I em cães normais e em algumas situações específicas como miocardiopatias, arritmias, dispneia e síndrome dilatação-torção gástrica e após exercícios prolongados (Herndon et al., 2002; Schobber et al., 2002b; Burgener et al., 2006; Fonfara et al., 2010).

Adin et al. (2006) mensuraram a cTnI em três diferentes analisadores - Biosite Triage Meter, Dade-Behring Stratus e Beckman-Counter Access AccuTnI -, pela metodologia de imunoensaio, sendo que a cTnI foi purificada e diluída em oito concentrações $-0,01 ; 0,1 ; 0,78$; 1,$66 ; 3,13 ; 6,25 ; 12,5$ e $25 \mathrm{ng} / \mathrm{mL}$ - de amostras provenientes de cães cardiopatas e normais. Esses autores observaram correlação entre as mensurações realizadas nos três analisadores. Isto valida os resultados obtidos neste estudo.

O valor médio de $\mathrm{cTnI}$ foi de $0,16 \pm 0,11 \mathrm{ng} / \mathrm{mL}$ e média no grupo 1 e de $0,20 \pm 0,11 \mathrm{ng} / \mathrm{mL}$, no grupo 2. Os valores de cTnI encontrados no grupo 1 assemelham-se aos referidos por Spratt et al. (2005), Adin et al. (2006), Burgener et al. (2006), Fonfara et al. (2010) em animais hígidos de raças, portes e idades variados. O critério de escolha dos cães de cada grupo baseou-se no exame eletrocardiográfico. Cães mais velhos são passíveis de apresentar deflexões do segmento ST e hipóxia-isquemia miocárdica, em razão da maior predisposição à injúria do endotélio das artérias coronárias e em decorrência das válvulo e miocardiopatias.

Nos animais do grupo 2 a distribuição do peso corpóreo foi mais homogênea do que nos do grupo 1, em razão da predominância de animais da raça Poodle, sete animais, e Cocker Spaniel, três animais no grupo 2, que juntos totalizaram $55 \%$ da amostra.

Na literatura consultada, não foram encontrados estudos, em cães, que associaram os níveis de cTnI com as deflexões do segmento ST. Sabe-se que um dos estímulos para a formação de vasos colaterais, a partir do tronco das coronárias, é a hipóxia-isquemia crônicas (Bonow, 2009). A partir dessa informação, poder-se-ia pensar que nos animais do grupo 2, com oxigenação miocárdica deficitária, houvesse estimulação para a formação de colaterais, suficientes para manter a integridade ou tornar mínimo o dano ao miócito, mas que não evitasse o distúrbio de 
repolarização. Burgener et al. (2006) detectaram em cães sacrificados devido à síndrome dilatação-torção-gástrica valores de cTnI de até $369 \mathrm{ng} / \mathrm{mL}$. Estes valores são típicos de lesões extensas e agudas do miocárdio, não passíveis de compensação pelo surgimento de vasos colaterais, diferente do que ocorreu com os cães do grupo 2. Sabe-se que as troponinas prestam-se mais ao diagnóstico de lesões agudas, como as obtidas nas lesões oclusivas (Ramos e Magalhães, 2000; Prosek et al., 2007) ou crônicas com maior comprometimento miocárdico - cTnI $>1 \mathrm{ng} / \mathrm{mL}$ - (Fonfara et al., 2010), aspectos que poderiam explicar, unidos à formação dos vasos colaterais, os resultados semelhantes obtidos nos grupos.

\section{CONCLUSÕES}

O kit de ensaio imunométrico por quimioluminescência humano, para a mensuração sérica de cTnI, pode ser utilizado na espécie canina, com a finalidade de monitorar possíveis danos ao miocárdio, em condições clínicas ou experimentais; os valores médios de cTnI obtidos em animais normais não podem ser considerados referenciais, em razão do tamanho da amostra do estudo, mas confirmam os resultados encontrados por outros autores; se não houver diferenças entre os animais, sugere-se que a cTnI não seja sensível na detecção de lesões miocárdicas ou que estas são mínimas.

\section{REFERÊNCIAS BIBLIOGRÁFICAS}

ADIN, D.B.; OYAMA, M.A.; SLEEPER, M.M. et al. Comparison of canine cardiac troponin I concentrations as determined by 3 analysers. $J$. Vet. Intern. Med., v.20, p.1136-1142, 2006.

BONAGURA, J.D. M-mode echocardiography basic principles. Vet. Clin. N. Am.: Small Animal Pract., v.12, p.299-319, 1983.

BONOW, R.O.; LIBBY, P.; ZIPES, D.P. Braunwald-Tratado de doenças cardiovasculares. São Paulo: Elsevier, 2009. 2432p.

BOON, J.A. The echocardiographic examination. In: BOON, J.A. Manual of Veterinary Echocardiography. Baltimore: Williams and Wilkins, 1998. p.151-260.
BURGENER, I.A.; KOVACEVIC, A.; MAULDIN, G.N. et al. Cardiac Troponins as Indicators of Acute Myocardial Damage in Dogs. J. Vet. Intern. Med., v.20, p.277-283, 2006.

DIAGNOSTIC Products Corporation. Troponin I. São Paulo: DPC, 2002.

FEITOSA, F.L.F.; CIARLINI, L.D.R.P.; CIARLINI, P.C. Quimiluminescência: princípio e aplicações. Rev. Ed. Cont., v.5, p.181-185, 2002.

FENG, Y.J.; CHEN, C.; FALLON, J.T. et al. Comparison of Cardiac Troponin I, Creatine Kinase-MB, and Myoglobin Injury in Swine Model. Clin. Chem., v.110, p.70-77, 1998.

FONFARA, S.; LOUREIRO, J.; SWIFT, S. et al. Cardiac troponin I as a marker for severity and prognosis of cardiac disease in dogs. Vet. J., v.184, p.334-339,2010.

FOX, P.R. Moléstias do Miocárdio. In: ETTINGER, S.J. Tratado de Medicina Interna Veterinária. São Paulo: Manole, 1992. p.11531189.

HERNDON, W.E; KITTLESON, M.D.; SANDERSON, K. et al. Cardiac Troponin I in Feline Hypertrophic Cardiomyopathy. J. Vet. Int. Med., v.16, p.558-564, 2002.

LOBETTI, R.; DVIR, E.; PEARSON, J. Cardiac Troponins in Canine Babesioses. J. Vet. Intern. Med., n.16, p.63-68, 2002.

MEYER, D.J.; HARVEY, J.W. Veterinary Laboratory Medicine. Philadelphia: WB. Saunders Company, 1998.

MOÏSE, N.S.; FOX, P.R. Echocardiography and doppler imaging. In: FOX, P.R.; SISSON, D.; MOÏSE, N.S. Textbook of canine and feline cardiology. 2.ed. Philadelphia: Saunders, 1999. p.130-171.

PROCAJLO, A.; ZBANYSZEK, M.; SOBIECH, P. et al. Troponin: a new marker in the diagnostics of muscle diseases in animals. J. Vet. Science. v.6, p.297-309, 2005.

PROSEK, R.; SISSON, D.D.; OYAMA, M.A. et al. Distinguishing cardiac and noncardiac dyspnea in 48 dogs using plasma atrial natriuretic factor, B-type natriuretic factor, endothelin, and cardiac troponin I. J. Vet. Intern. Med., v.21, p.238-242, 2007. 
RAMOS, R.F.; MAGALHÃES，H.M. Infarto agudo do miocárdio: novos métodos de diagnóstico e prognóstico. In: TIMERMAN, A.; CÉSAR, L.A.M.(Ed.). Manual de Cardiologia (SOCESP). São Paulo: Atheneu, 2000. p.147151.

RISHNIW, M.; BARR, S.C.; SIMPSON, K.W. et al. Cloning and sequencing of the canine and feline cardiac troponin I genes. Am. J. Vet. Res., v.65, p.53-58, 2004

ROBBINS, S.L.; KUMAR, V.; COTRAN, R.S. Patologia Estrutural e Funcional. 6.ed. Rio de Janeiro: Guanabara Koogan, 2001.780p.

SCHOBBER, K.; KIRBACH, B.; CORNAND, C. et al. Diagnostische und differenzialdiagnostische Wertigkeit zirkulierender kardialer Troponine bei Hund und Katze. Tierärztl. Prax, v.30, p.327331, 2002a.

SCHOBBER, K.; KIRBACH, B.; CORNAND, C. et al. Serum cardiac troponin I and cardiac troponin $\mathrm{T}$ concentrations in dogs with gastric dilatation-volvulus. J. Am. Vet. Med. Assoc., v.221, p.381-388, 2002b.
SPRATT, D.P.; MELLANBY, R.J; DRURY, N. et al. Cardiac troponin I: evaluation I of a biomarker for the diagnosis oh heart disease in the dog. J. Small Anim. Pract, v.46, p.113-114, 2005.

THOMAS, W.P. Myocardial disease of the dog. In: BONAGURA, J.D. (Ed.). Cardiology. New York: Churchill Livingstone, 1987. p.117-156.

TILLEY, P.L. ST segment abnormalities. In:__Essentials of Canine and Feline Eletrocardiography: Interpretation and Treatment. USA: Lea \& Febiger, 1992. p.84-87.

TILLEY, L.P.; JUNIOR, F.W.K.S. Consulta Veterinária em 5 minutos. São Paulo: Manole, 2008.

WEEKS, I. Chemiluminescence Immunoassay. In: PRICE, C.P. Principles and practice of immunoassay. London: MacMilan, 1997. p.427442. 